\title{
Network Equilibrium of Heterogeneous Congestion Control Protocols
}

\author{
Ao Tang Jiantao Wang Steven H. Low \\ EAS Division, California Institute of Technology \\ Mung Chiang \\ EE Department, Princeton University
}

\begin{abstract}
When heterogeneous congestion control protocols that react to different pricing signals share the same network, the resulting equilibrium may no longer be interpreted as a solution to the standard utility maximization problem. We prove the existence of equilibrium under mild assumptions. Then we show that multi-protocol networks whose equilibria are locally non-unique or infinite in number can only form a set of measure zero. Multiple localty unique equilibria can arise in two ways. First, unlike in the single-protocol case, the set of bottleneck links can be non-unique with heterogeneous protocols even when the routing matrix has full row rank. The equilibria associated with different sets of bottleneck links are necessarily distinct. Second, even when there is a unique set of bottleneck links, network equilibrium can still be non-unique, but is always finite and odd in number. They cannot all be locally stable unless it is globally unique. Finally, we provide various sufficient conditions for glohal uniqueness. Numerical exumples are used throughout the paper to illustrate these results.
\end{abstract}

Index Terms-Congestion control, Heterogeneous protocols, Multiprotocol networks, Equilibrium analysis

\section{INTRODUCTION}

\section{A. Motivation}

Congestion control protocols have been modelled as distributed algorithms for utility maximization, e.g., [9], [13], [20], [28], [10], [12]. With the exception of a few limited analysis on very simple topologies [19], [11], [12], existing literature generally assumes that all sources are homogeneous in that, even though they may control their rates using different algorithms, they all adapt to the same type of congestion signals, e.g., all react to loss probabilities, as in TCP Reno, or all to queueing delay, as in TCP Vegas or FAST [8]. When sources with heterogeneous protocols that react to different congestion signals share the same network, the current duality framework is no longer applicable. With more congestion control protocols being proposed and ideas of using congestion signals other than packet losses, including explicit feedbacks, being developed in the networking community, we need a mathematically rigorous framework to understand the behavior of large-scale networks with heterogeneous protocols. The purpose of this paper is to propose such a framework.

Our emphasis is on general networks with multiple sources and links that use a large class of algorithms to adapt their rates and congestion prices. Often, interesting and counterintuitive behaviors arise only in a network setting wherc sources interact through shared links in intricate and surprising ways, e.g., [26]. Such behaviors are absent in single-link models and are usually hard to discover or explain without a fundamental understanding of the underlying structure. Given the scale and heterogeneity of the Internet, it is conceivable that such behaviors are more common than we realize, but remain difficult to measure due to the complexity of the infrastructure and our inability to monitor it closely. A mathematical framework thus becomes indispensable in exploring structures, clarifying ideas, and suggesting directions. Some of the theoretical predictions in this paper have already been demonstrated experimentally in [25].

\section{B. Summary}

A congestion control protocol generally takes the form

$$
\begin{aligned}
& \dot{p}_{i}=g_{I}\left(\sum_{j: l \in L(j)} x_{j}(t), p_{l}(t)\right) \\
& \dot{x}_{j}=f_{j}\left(x_{j}(t), \sum_{l \in L(j)} m_{l}^{j}\left(p_{l}(t)\right)\right)
\end{aligned}
$$

Here, $L(j)$ denotes the set of links used by source $j$, and $g_{l}(\cdot)$ models a queue management algorithm that updates the price $p_{i}(t)$ at link $l$, often implicitly, based on its current value and the sum of source rates $x_{j}(t)$ that traverse link $l$. The prices may represent loss probabilities, queueing delays, or quantities explicitly calculated by the links and fed back to the sources. The function $f_{j}(\cdot)$ models a TCP algorithm that adjusts the transmission rate $x_{j}(t)$ of source $j$ based on its current value and the sum of "effective prices" $m_{i}^{j}\left(p_{l}(t)\right)$ in its path. The effective prices $m_{l}^{j}\left(p_{l}(t)\right)$ are functions of the link prices $p_{l}(t)$, and the functions $m_{l}^{j}$ in general can vary depending on the links and sources.

When all algorithms use the same pricing signal, i.e., $m_{l}^{j}=m_{l}$ are the same for all sources $j$, the equilibrium properties of (1)-(2) turn out to be very simple. Indeed, under mild conditions on $g_{l}(\cdot)$ and $f_{j}(\cdot)$, the equilibrium of (1)(2) exists and is unique [12]. This is proved by identifying the equilibrium of (1)-(2) with the unique solution of the utility maximization problem defined in [9] and its Lagrange dual problem [13]. Here, the equilibrium prices $p l$ play the role of Lagrange multipliers, one at each link. This utility maximization problem thus provides a simple and complete characterization of the equilibrium of a single-protocol network. 
When heterogeneous algorithms that use different pricing signals share the same network, i.e., $m_{l}^{j}$ are different for different sources $j$, the situation is much more complicated. For instance, when TCP Reno and TCP Vegas or FAST share the same network, neither loss probability nor queueing delay can serve as the Lagrange multiplier at the link, and (1)-(2) can no longer be interpreted as solving the standard utility maximization problem. Basic questions, such as the existence and uniqueness of equilibrium, its local and global stability, need to be re-examined.

In the multi-protocol case, we prove that equilibrium still exists, under mild conditions, despite the lack of an underlying concave optimization problem (Section III). In contrast to the single-protocol case, even when the routing matrix has full row rank, there can be uncountably many equilibria (Example 1 in Section IV) and the set of bottleneck links can be nonunique (Example 2 in Section IV). However, we prove that almost all networks have finitely many equilibria and they are necessarily locally unique (Section IV). Non-uniqueness can arise in two ways. First, the equilibria associated with different sets of bottleneck links are always distinct. Second, the number of equilibria associated with each set of bottleneck links can be more than one, though always odd (Section IV). Moreover, these equilibria cannot all be locally stable unless the equilibrium is globally unique (Section V). Finally, we provide three additional sufficient conditions for global uniqueness of network equilibrium (Section V). The first condition generalizes the full-rank condition on $R$ for global uniqueness from single-protocol networks to multi-protocol networks. The second condition guarantees global uniqueness when the price mapping functions $m_{l}^{j}=m^{j}$ are linear and link-independent. The last condition implies global uniqueness of a class of linear networks. Throughout the paper, we provide numerical examples to illustrate equilibrium properties or how a theorem can be applied. In [25], we demonstrate experimentally the phenomenon of multiple equilibria using TCP Reno and TCP Vegas/FAST in ns-2 simulator/Dummynet testbed.

Our formulation is close to the general equilibrium theory [17] in economics from which we borrow some ideas and techniques. See [4], [6], [7], [22], [23], [27], [3], [5], [16], [1] for related works. The main mathematical tools used in this paper are the Nash theorem in game theory [21], [2], which is an application of Kakutani's generalized fixed point theorem, and results from differential topology, especially the PoincareHopf Index Theorem [18]. They are used to prove existence and study uniqueness of network equilibrium, respectively.

\section{MODEL}

\section{A. Notation}

A network consists of a set of $L$ links, indexed by $l=$ $1, \ldots, L$, with finite capacities $c_{l}$. We often abuse notation and use $L$ to denote both the number of links and the set $L=\{1, \ldots, L\}$ of links. Each link has a price $p_{l}$ as its congestion measure. There are $J$ different protocols indexed by superscript $j$, and $N^{j}$ sources using protocol $j$, indexed by $(j, i)$ where $j=1, \ldots, J$ and $i=1, \ldots, N^{j}$. The total number of sources is $N:=\sum_{j} N^{j}$.

The $L \times N^{j}$ routing matrix $R^{j}$ for type $j$ sources is defined by $R_{l i}^{j}=1$ if source $(j, i)$ uses $\operatorname{link} l$, and 0 otherwise. The overall routing matrix is denoted by

$$
R=\left[\begin{array}{llll}
R^{1} & R^{2} & \cdots & R^{J}
\end{array}\right]
$$

All type $j$ sources react to "effective prices" $p_{i}^{j}$ at links $l$ in their paths. The effective price $p_{l}^{j}$ is determined by the link price $p_{l}$ through a price mapping function

$$
p_{l}^{j}=m_{l}^{j}\left(p_{l}\right)
$$

which can depend on both the link and the protocol type. By specifying function $m_{l}^{j}$, we can let the link feed back different congestion signals to sources using different protocols, for example, Reno with packet losses and Vegas with queueing delay. Let $m^{j}(p)=\left(m_{l}^{j}\left(p_{l}\right), l=1, \ldots L\right)$ and $m(p)=$ $\left(m^{j}\left(p_{i}\right), j=1, \ldots J\right)$.

The aggregate prices for source $(j, i)$ is defined as

$$
q_{i}^{j}=\sum_{l} R_{l i}^{j} p_{l}^{j}=\sum_{l} R_{l i}^{j} m_{l}^{j}\left(p_{l}\right)
$$

Let $q^{j}=\left(q_{i}^{j}, i=1, \ldots, N^{j}\right)$ and $q=\left(q^{j}, j=1 \ldots, J\right)$ be vectors of aggregate prices. Then $q^{j}=\left(R^{j}\right)^{T} m^{j}(p)$ and $q=R^{T} m(p)$ where ${ }^{T}$ denotes matrix transpose.

Let $x^{j}$ be a vector with the rate $x_{i}^{j}$ of source $(j, i)$ as its $i$ th entry, and $x$ be the vector of $x^{j}$

$$
x=\left[\left(x^{1}\right)^{T},\left(x^{2}\right)^{T}, \ldots,\left(x^{J}\right)^{T}\right]^{T}
$$

Source $(j, i)$ has a utility function $U_{i}^{j}\left(x_{i}^{j}\right)$ that is strictly concave increasing in its rate $x_{i}^{j}$. Let $U=\left(U_{i}^{j}, i=1, \ldots, N^{j}, j=\right.$ $1, \ldots, l)$.

In general, if $z_{k}$ is defined, then $z$ denotes the (column) vector $z=\left(z_{k}, \forall k\right)$. Other notations will be introduced later when they are encountered. We call $(c, m, R, U)$ a network.

\section{B. Network equilibrium}

A network is in equilibrium, or the link prices $p$ and source rates $x$ are in equilibrium, when each source $(j, i)$ maximizes its net benefit (utility minus bandwidth cost), and the demand and supply of bandwidth at each link are balanced. Formally, a network equilibrium is defined as follows.

Given any prices $p$, we assume in this paper that the source rates $x_{i}^{j}$ are uniquely determined by

$$
x_{i}^{j}\left(q_{i}^{j}\right)=\left[\left(U_{i}^{j}\right)^{\prime-1}\left(q_{i}^{j}\right)\right]^{+}
$$

where $\left(U_{i}^{j}\right)^{\prime}$ is the derivative of $U_{i}^{j}$, and $\left(U_{i}^{j}\right)^{t-1}$ is its inverse which exists since $U_{i}^{j}$ is strictly concave. Here $[z]^{+}=$ $\max \{z, 0\}$. This implies that the source rates $x_{i}^{j}$ uniquely solve

$$
\max _{z \geq 0} U_{i}^{j}(z)-z q_{i}^{j}
$$

As we will see, under the assumptions in this paper, $\left(U_{i}^{j}\right)^{\prime-1}\left(q_{i}^{j}\right)>0$ for all the prices $p$ that we consider, and 
hence we can ignore the projection $[\cdot]^{+}$and assume without loss of generality that

$$
x_{i}^{j}\left(q_{i}^{j}\right)=\left(U_{i}^{j}\right)^{\prime-1}\left(q_{i}^{j}\right)
$$

As usual, we use $x^{j}\left(q^{j}\right)=\left(x_{i}^{j}\left(q_{i}^{j}\right), i=1, \ldots, N^{j}\right)$ and $x(q)=\left(x^{j}\left(q^{j}\right), j=1, \ldots, J\right)$ to denote the vector-valued functions composed of $x_{i}^{j}$. Since $q=R^{T} m(p)$, we often abuse notation and write $x_{i}^{j}(p), x^{j}(p), x(p)$.

Define the aggregate source rates $y(p)=\left(y_{l}(p), l=\right.$ $1, \ldots, L)$ at links $l$ as:

$$
y^{j}(p)=R^{j} x^{j}(p), \quad y(p)=R x(p)
$$

In equilibrium, the aggregate rate at each link is no more than the link capacity, and they are equal if the link price is strictly positive. Formally, we call $p$ an equilibrium price, a network equilibrium, or just an equilibrium if it satisfies (from (3)-(5))

$$
P(y(p)-c)=0, \quad y(p) \leq c, \quad p \geq 0
$$

where $P$ is a diagonal matrix defined as $P:=\operatorname{diag}\left(p_{l}\right)$. The goal of this paper is to study the existence and uniqueness properties of network equilibrium specified by (3)-(6). Let $E$ be the equilibrium set:

$$
E=\left\{p \in \Re_{+}^{L} \mid P(g(p)-c)=0, y(p) \leq c\right\}
$$

For future use, we now define an active constraint set and the Jacobian for links that are actively constrained. Fix an equilibrium price $p^{*} \in E$. Let the active constraint set $\hat{L}=$ $\hat{L}\left(p^{*}\right) \subseteq L$ (with respect to $p^{*}$ ) be the set of links $l$ at which $p_{l}^{*}>0$. Consider the reduced system that consists only of links in $\hat{L}$, and denote all variables in the reduced system by $\hat{c}, \hat{p}, \hat{y}$, etc. Then, since $y_{l}(p)=c_{l}$ for every $l \in \hat{L}$, we have $\hat{y}(\hat{p})=\hat{c}$. Let the Jacobian for the reduced system be $\hat{\mathbf{J}}(\hat{p})=\partial \hat{y}(p) / \partial \hat{p}$. Then

$$
\hat{\mathbf{J}}(\bar{p})=\sum_{j} \hat{R}^{j} \frac{\partial x^{j}(p)}{\partial \hat{q}^{j}}\left(\hat{R}^{j}\right)^{T} \frac{\partial \hat{m}^{j}(p)}{\partial \hat{p}}
$$

where

$$
\begin{aligned}
\frac{\partial x^{j}}{\partial \hat{q}^{j}} & =\operatorname{diag}\left(\left(\frac{\partial^{2} U_{i}^{j}}{\partial\left(x_{i}^{j}\right)^{2}}\right)^{-1}\right) \\
\frac{\partial \hat{m}^{j}}{\partial \hat{p}} & =\operatorname{diag}\left(\frac{\partial \hat{m}_{l}^{j}}{\partial \hat{p}_{l}}\right)
\end{aligned}
$$

and all the partial derivatives are evaluated at the generic point $\hat{p}$.

\section{Current theory: $J=1$}

In this subsection, we briefly review the current theory for the case where there is only one protocol, i.e., $J=1$, and explain why it cannot be directly applied to the case of heterogeneous protocols.
When all sources react to the same price, then the equilibrium described by (3)-(6) is the unique solution of the following utility maximization problem defined in [9]:

$$
\begin{aligned}
\max _{x \geq 0} & \sum_{i} U_{i}\left(x_{i}\right) \\
\text { subject to } & R x \leq c
\end{aligned}
$$

where we have omitted the superscript $j=1$. The strict concavity of $U_{i}$ guarantees the existence and uniqueness of the optimal solution of (11)-(12). The basic idea to relate the utility maximization problem (11)-(12) to the equilibrium equations (3)-(6) is to examine the dual of the utility maximization problem, and interpret the effective price $m_{l}\left(p_{l}\right)$ as a Lagrange multiplier associated with each link capacity constraint (see, e.g., [13], [20], [12]). As long as $m_{l}\left(p_{l}\right) \geq 0$ and $m_{l}(0)=0$, one can replace $p_{l}$ in $(6)$ by $m_{l}\left(p_{l}\right)$. The resulting equation together with (3)-(5) provides the necessary and sufficient condition for $x_{i}(p)$ and $m_{l}\left(p_{l}\right)$ to be primal and dual optimal respectively.

This approach breaks down when there are $J>1$ types of prices because there cannot be more than one Lagrange multiplier at each link. In general, an equilibrium no longer maximizes aggregate utility, nor is it unique. However, as shown in the rlext section, existence of equilibrium is still guaranteed under the following mild assumptions.

A1: Utility functions $U_{i}^{j}$ are strictly concave increasing, and twice continuously differentiable in their domains. Price mapping functions $m_{l}^{j}$ are continuously differentiable in their domains and strictly increasing with $m_{l}^{j}(0)=0$.

A2: For any $\epsilon>0$, there exists a number $p_{\max }$ such that if $p_{l}>p_{\max }$ for link $l$, then

$$
x_{i}^{j}(p)<\epsilon \text { for all }(j, i) \text { with } R_{l i}^{j}=1
$$

These are mild assumptions. Concavity and monotonicity of utility functions are often assumed in network pricing for elastic traffic. The assumption on $m_{l}^{j}$ preserves the relative order of prices and maps zero price to zero effective price. Assumption A2 says that when $p_{l}$ is high enough, then every source going through link $l$ has a rate less than $\epsilon$.

\section{EXISTENCE OF EQUILIBRIUM}

In this section, we prove the existence of network equilibrium. We start with a lemma that bounds the equilibrium prices.

Lemma 1. Suppose $A 1$ and $A 2$ hold. Given a network $(c, m, R, U)$, there is a scalar $p_{\max }$ that upper bounds any equilibrium price $p$. i.e., $p_{l} \leq p_{\max }$ for all $l$.

Proof. Choose $\epsilon=\min _{l} c_{l} / N$, and let $p_{\max }$ be the corresponding scalar in A2. Suppose that there exists an equilibrium price $p$ and a link $l$, such that $p_{l}>p_{\max }$. A2 implies that the aggregate equilibrium rate at link $l$ satisfies

$$
\sum_{j} \sum_{i} R_{l i}^{j} x_{i}^{j}(p)<N \epsilon=\min _{l} c_{l}
$$

Therefore, we get a link with $p_{l}>0$ but not fully utilized. It contradicts the equilibrium condition (6). 
The following theorem asserts the existence of equilibrium for a multi-protocol network.

Theorem 2. Suppose AI and A2 hold. There exists an equilibrium price $p^{*}$ for any network $(c, m, R, U)$.

Proof. Let $p_{\max }$ be the scalar upper bound in Lemma 1. For any $p \in\left[0, p_{\max }\right]^{L}$, define a vector function

$$
F(p):=R x(p)-c
$$

For any link $l$, let

$$
p_{-i}:=\left(p_{1}, \ldots, p_{l-1}, p_{l+1}, \ldots p_{L}\right)^{T}
$$

Then we may write $F(p)$ as $F\left(p_{l}, p_{-l}\right)$. Define function $h_{l}$ as

$$
h_{l}\left(p_{l}, p_{-l}\right):=-F_{l}^{2}\left(p_{l}, p_{-l}\right)
$$

We claim that $h_{l}\left(p_{l}, p_{-l}\right)$ is a quasi-concave function in $p_{l}$ for any fixed $p_{-l}$. By the definition of quasi-concavity in [21], we only need to check that the set

$$
A_{l}:=\left\{p_{l} \mid h_{l}\left(p_{i}, p_{-l}\right) \geq a\right\}
$$

is convex for all $a \in \Re$. If $a>0$, clearly $A_{l}=\emptyset$ by (14), When $a \leq 0$, the set $A_{l}$ can be rewritten as

$$
A_{l}=\left\{p_{l} \mid-\sqrt{|a|} \leq F_{l}\left(p_{l}, p_{-l}\right) \leq \sqrt{|a|}\right\}
$$

Since $F_{l}\left(p_{l}, p_{-l}\right)$ is a non-increasing function in $p_{l}$ for any fixed $p_{-l}$, the set $A_{l}$ is convex. Therefore $h_{l}\left(p_{l}, p_{-l}\right)$ is quasiconcave in $p_{l}$.

Since $\left[0, p_{\max }\right]$ is a nonempty compact convex set, by the theorem of Nash [21], the quasi-concavity of $h_{l}\left(p_{l}, p_{-l}\right)$ guarantees that there exists a $p^{*} \in\left[0, p_{\max }\right]^{L}$ such that for all $l \in\{1,2 \ldots L\}$

$$
p_{l}^{*}=\arg \max _{p_{l} \in\left[0, p_{\max }\right]} h_{l}\left(p_{l}, p_{-l}^{*}\right)
$$

We now argue that, for all $l$, either 1) $F_{l}\left(p^{*}\right)=0$, or 2) $F_{l}\left(p^{*}\right)<0$ and we can take $p_{l}^{*}=0$. These conditions imply (6), and hence $p^{*}$ is an equilibrium price.

Case 1: $F_{l}\left(0, p_{-1}^{*}\right)>0$. Since $U_{i}^{j}$ is strictly concave, $F_{l}\left(p_{l}, p_{-l}^{*}\right)$ is nondecreasing ${ }^{\mathrm{f}}$ in $\left[0, p_{\max }\right]$. Moreover, the proof of Lemma 1 shows that $F_{l}\left(p_{\max }, p_{*-1}^{*}\right)<0$. Therefore, there exists a point $p_{l}^{*}$ in $\left[0, p_{\max }\right]$ where $\cdot F_{l}\left(p_{l}, p_{-l}^{*}\right)=0$. This $p_{l}^{*}$ maximizes $h_{l}\left(p_{l}, p_{-l}^{*}\right)$.

Case 2: $F_{l}\left(0, p_{-l}^{*}\right) \leq 0$. Since $F_{l}\left(p_{l}, p_{-l}^{*}\right)$ is a non-increasing function in $p_{l}$, we have that

$$
F_{l}\left(p_{l}, p_{-l}^{*}\right) \leq 0 \text { for all } p_{l} \in\left[0, p_{\max }\right]
$$

If $-c_{l}<F_{l}\left(0, p_{-l}^{*}\right) \leq 0$, then $F_{l}\left(p_{l}, p_{-l}^{*}\right)$ and $h_{l}\left(p_{l}, p_{-l}^{*}\right)$ are strictly decreasing in $p_{l}$ and hence

$$
p_{l}^{*}=\arg \max _{p_{l} \in\left[0, p_{\text {max }}\right]} h_{l}\left(p_{l}, p_{-l}^{*}\right)=0
$$

Otherwise we have $F_{l}\left(0, p_{-l}^{*}\right)=-c_{l}$ from (13). In this situation, all $x_{i}^{j}$ going through link $l$ are zero, and hence we can set $p_{l}^{*}=0$ without affecting any other prices. More precisely, a (possibly) new price vector $\tilde{p}$ with $\tilde{p}_{l}=0$ and

\footnotetext{
${ }^{1} F_{l}^{*}\left(p_{l}, p_{-l}^{*}\right)$ is strictly increasing unless some $x_{i}(p)$ becomes zero.
}

$\tilde{p}_{k}=p_{k}^{*}$ for $k \neq l$ is also a Nash equilibrium that maximizes $h_{k}\left(p_{k}, \tilde{p}_{-k}\right)$ for $k=1, \ldots, L$.

Thus we have proved that, for $l=1, \ldots, L$,

$$
p_{l}^{*} F_{l}\left(p_{l}^{*}, p_{-l}^{*}\right)=0, \quad F_{l}\left(p_{l}^{*}, p_{-l}^{*}\right) \leq 0, p^{*} \geq 0
$$

which is $(6)$.

\section{LOCAL UNIQUENESS OF EQUILIBRIUM}

Theorem 2 guarantees the existence of network equilibrium. We now study its uniqueness properties.

In a single-protocol network, if the routing matrix $R$ has full row rank, then there is a unique active constraint set $\hat{L}$ and a unique equilibrium price $p$ associated with it [20]. If $R$ does not have full row rank, then equilibrium prices $p$ may be nonunique but the equilibrium rates $x(p)$ are still unique since the utility functions are strictly concave. This also implies that the set of links $l$ with $y_{l}(p)=c_{l}$ is the same for any equilibrium price $p$, though the active constraint sets where $p_{l}>0$ may not be unique.

In contrast, the active constraint set in a multi-protocol network can be non-unique even if $R$ has full row rank. Clearly, the equilibrium prices associated with different active constraint sets are different. Moreover, there can be multiple equilibrium prices associated with the same active constraint set, as we prove below.

We start with two examples of multiple equilibria. Example $I$ has a unique active constraint set, and yet it exhibits uncountably many equilibria. This example is the basis for other examples in this paper. Example 2 has multiple active constraint sets but there is a unique (but different) equilibrium associated with each active constraint set. We then introduce regular networks whose equilibrium prices are locally unique and characterize them

\section{A. Two examples of multiple equilibria}

Example 1: unique active constraint set but uncountably many equilibria

In this example, we assume all the sources use the same utility function defined as

$$
U_{i}^{j}\left(x_{i}^{j}\right)=-\frac{1}{2}\left(1-x_{i}^{j}\right)^{2}
$$

Then the cquilibrium rates $x^{j}$ of type $j$ sources are determined by the equilibrium prices $p$ as

$$
x^{j}(p)=1-\left(R^{j}\right)^{T} m^{j}(p)
$$

where 1 is a vector of appropriate dimension whose entries are all 1s. We use linear price mapping functions:

$$
m^{\dot{j}}(p)=K^{j} p
$$

where $K^{j}$ are $L \times L$ diagonal matrices. Then the equilibrium rate vector of type $j$ sources can be expressed as

$$
x^{j}(p)=1-\left(R^{j}\right)^{T} K^{j} p
$$


When only links with strictly positive equilibrium prices are included in the model, we have

$$
y(p)=\sum_{j} R^{j} x^{j}(p)=c
$$

Substituting in $x^{j}(p)$ yields

$$
\sum_{j} R^{j}\left(R^{j}\right)^{T} K^{j} p=\sum_{j} R^{j} \mathbf{1}-c
$$

which is a linear equation in $p$ for given $R^{j}, K^{j}$, and $c$. It has a unique solution if the determinant is nonzero, but has no or multiple solutions if

$$
\operatorname{det}\left(\sum_{i} R^{i}\left(R^{i}\right)^{T} K^{i}\right)=0
$$

When $J=1$, i.e., when there is only one protocol and $R$ (which is the same as $R^{1}$ here) has full row rank, $\operatorname{det}\left(R^{1}\left(R^{1}\right)^{T} K^{1}\right)>0$ since both $R^{1}\left(R^{1}\right)^{T}$ and $K^{1}$ are positive definite. In this case, there is a unique equilibrium price vector.

When $J=2$, there are networks whose determinants are zero that have uncountably many equilibria. See Example 5 in the Appendix for one where $R$ does not have full row rank. We provide here an example with $J=3$ where $R$ still has full row rank.

The network is shown in Figure 1 with three unit-capacity links, $c_{l}=1$. There are three different protocols with the

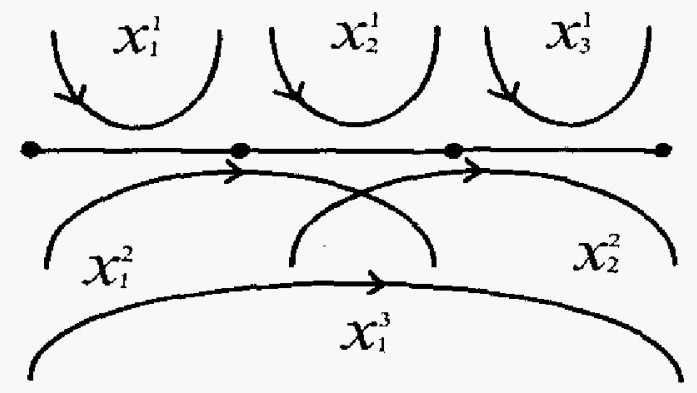

Fig. 1. Example 1: uncountably many equilibria.

corresponditg routing matrices

$$
R^{1}=I, \quad R^{2}=\left[\begin{array}{lll}
1 & 1 & 0 \\
0 & 1 & 1
\end{array}\right]^{T}, \quad R^{3}=(1,1,1)^{T^{\prime}}
$$

The linear mapping functions are given by

$$
K^{-1}=I, \quad K^{2}=\operatorname{diag}(5,1,5), \quad K^{3}=\operatorname{diag}(1,3,1)
$$

It is easy to calculate that

$$
\sum_{i=1}^{3} R^{i}\left(R^{i}\right)^{T} K^{-i}=\left[\begin{array}{lll}
7 & 4 & 1 \\
6 & 6 & 6 \\
1 & 4 & 7
\end{array}\right]
$$

which has determinant 0 . Using the utility function defined in (15), we can check that all the following are equilibrium prices

$$
\begin{aligned}
& p_{1}^{1}=p_{3}^{1}=1 / 8+\epsilon \\
& p_{2}^{1}=1 / 4-2 \epsilon \text { where } \epsilon \in[0,1 / 24]
\end{aligned}
$$

The corresponding rates are

$$
\begin{aligned}
x_{1}^{1}=x_{3}^{1}=7 / 8-\epsilon & x_{2}^{1}=3 / 4+2 \epsilon \\
x_{1}^{2}=x_{2}^{2}=1 / 8-3 \epsilon & x_{1}^{3}=4 \epsilon
\end{aligned}
$$

All capacity constraints are tight with these rates. Since there is an one-link flow at every link, the active constraint set is unique and contains every link. Yet there are uncountably many equilibria.

\section{Example 2: multiple active constraint sets each with a unique equilibrium}

Consider the symmetric network in Figure 2 with 3 flows. There are two protocols in the network with the following

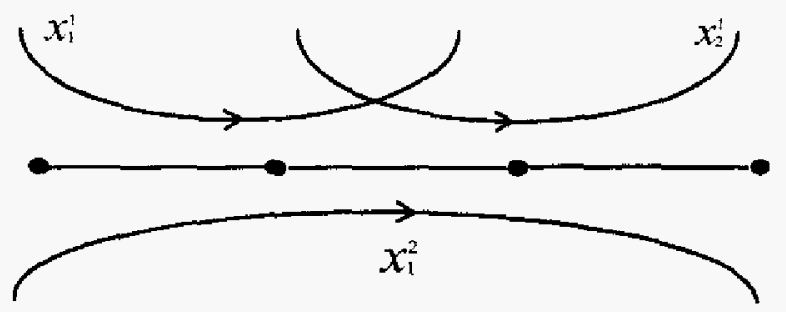

Fig. 2. Example 2: two active constraint sets.

routing matrices

$$
R^{\mathrm{i}}=\left[\begin{array}{ll}
1 & 0 \\
1 & 1 \\
0 & 1
\end{array}\right], \quad R^{2}=(1,1,1)^{T}
$$

Flows $(1,1)$ and $(1,2)$ have identical utility function $V^{1}$ and source rate $x^{1}$, and flow $(2,1)$ has a utility function $U^{2}$ and source rate $x^{2}$.

Links 1 and 2 both have capacity $c_{1}$ and price mapping functions $m_{1}^{1}(p)=p$ and $m_{1}^{2}(p)$ for protocols 1 and 2 respectively. Link 2 has capacity $c_{2}$ and price mapping functions $m_{2}^{1}(p)=p$ and $m_{2}^{2}(p)$.

The following theorem provides a sufficient condition for multiple equilibria [25].

Theorem 3. Suppose assumption AI holds. The network shown in Figure 2 has two equilibria provided:

1) $c_{1}<c_{2}<2 c_{1}$

2) for $j=1,2,\left(U^{j}\right)^{\prime}\left(x^{j}\right) \rightarrow \vec{p}^{j}$ possibly $\infty$, if and only if $x^{j} \rightarrow 0$.

3) for $l=1,2, m_{l}^{2}\left(p_{l}\right) \rightarrow \bar{p}^{2}$ as $p_{l} \rightarrow \bar{p}^{1}$, and satisfy

$$
\begin{array}{r}
2 m_{1}^{2}\left(\left(U^{1}\right)^{\prime}\left(c_{2}-c_{1}\right)\right)<\left(U^{2}\right)^{\prime}\left(2 c_{1}-c_{2}\right) \\
<m_{2}^{2}\left(\left(U^{I}\right)^{\prime}\left(c_{2}-c_{1}\right)\right)
\end{array}
$$

Proof: We first claim that, if $c_{1}<c_{2}$ and $\left(U^{2}\right)^{\prime}\left(2 c_{1}-c_{2}\right)>$ $2 m_{1}^{2}\left(\left(U^{1}\right)^{\prime}\left(c_{2}-c_{1}\right)\right)$, then there is an equilibrium point where only links 1 and 3 are saturated and link 2 is not. In this case the equilibrium price for link 2 is $p_{2}=0$ and, by symmetry, those for links 1 and 3 are both $p_{1}$. Such an equilibrium, if exists, is defined by the following equations:

$$
\begin{aligned}
\left(U^{1}\right)^{\prime}\left(x^{1}\right) & =p_{1} & & \left(U^{2}\right)^{\prime}\left(x^{2}\right)-2 m_{1}^{2}\left(p_{1}\right) \\
x^{1}+x^{2} & =c_{1} & & 2 x^{1}+x^{2}<c_{2}
\end{aligned}
$$


Eliminating $x^{2}$ and $p_{1}$, the above equations are reduced to:

$$
\begin{aligned}
\left(U^{2}\right)^{\prime}\left(c_{1}-x^{1}\right) & =2 m_{1}^{2}\left(\left(U^{1}\right)^{\prime}\left(x^{1}\right)\right) \\
x^{1} & <c_{2}-c_{1}
\end{aligned}
$$

An equilibrium exists if and only if (16)-(17) has a nonnegative solution for $x^{1}$. We now show that (16)-(17) indeed admits a unique solution $x^{*}>0$ under the hypothesis of the theorem.

When $x^{1}=0$, we have

$$
\left(U^{2}\right)^{\prime}\left(c_{1}-x^{1}\right)=\left(U^{2}\right)^{\prime}\left(c_{1}\right)<\bar{p}^{2} \leq 2 \bar{p}^{2}=2 m_{1}^{2}\left(\left(U^{2}\right)^{\prime}(0)\right)
$$

The inequality and the last equality have made multiple use of conditions 2 and 3 of the theorem. On the other hand, when $x^{1}=c_{2}-c_{1}$, we have $U_{2}^{\prime}\left(2 c_{1}-c_{2}\right)>2 m_{1}^{2}\left(U_{1}^{\prime}\left(c_{2}-c_{1}\right)\right)$ by condition 3 . Since all functions here are continuous, $\left(U^{j}\right)^{\prime}$ are strictly decreasing, and $m_{l}^{2}$ are strictly increasing, there exists a unique $0<x^{*}<c_{2}-c_{1}$ such that $\left(U^{2}\right)^{\prime}\left(c_{1}-x^{*}\right)=$ $2 m_{1}^{2}\left(\left(U^{1}\right)^{\prime}\left(x^{*}\right)\right)$.

We next claim that, if $c_{2}<2 c_{1}$ and $\left(U^{2}\right)^{\prime}\left(2 c_{1}-c_{2}\right)<$ $m_{2}^{2}\left(\left(U^{1}\right)^{\prime}\left(c_{2}-c_{1}\right)\right)$, then there is an equilibrium point where only link 2 is saturated and links 1 and 3 are not. In this case $p_{1}=p_{3}=0$, and the following equations determine such an equilibrium:

$$
\begin{array}{rl}
\left(U^{1}\right)^{\prime}\left(x^{1}\right)=p_{2} & \left(U^{2}\right)^{\prime}\left(x^{2}\right)=m_{2}^{2}\left(p_{2}\right) \\
x^{1}+x^{2}<c_{1} & 2 x^{1}+x^{2}=c_{2}
\end{array}
$$

Eliminating $x^{2}$ and $p_{2}$, the equilibrium is specified by

$$
\begin{aligned}
\left(U^{2}\right)^{\prime}\left(c_{2}-2 x^{1}\right) & =m_{2}^{2}\left(\left(U^{1}\right)^{\prime}\left(x^{1}\right)\right) \\
x^{1} & >c_{2}-c_{1}
\end{aligned}
$$

When $x^{1}=c_{2}-c_{1}$, we have

$$
\left(U^{2}\right)^{\prime}\left(c_{2}-2 x^{1}\right)=\left(U^{2}\right)^{\prime}\left(2 c_{1}-c_{2}\right)<m_{2}^{2}\left(\left(U^{1}\right)^{\prime}\left(x^{1}\right)\right)
$$

by condition 3 . When $x^{1}=c_{2} / 2$,

$$
\left(U^{2}\right)^{\prime}\left(c_{2}-2 x^{1}\right)=\left(U^{2}\right)^{\prime}(0)=\bar{p}^{2}>m_{2}^{2}\left(\left(U^{1}\right)^{\prime}\left(x^{1}\right)\right)
$$

where we have used conditions 2 and 3. Hence, again, there is a unique $x^{*}$ that satisfies (18)-(19). Moreover, from (17) and (19), the two equilibria are distinct.

Remark: TCP Reno, which reacts to loss probability, and TCP Vegas/FAST, which reacts to delay, have utility functions that satisfy the conditions in the theorem. Two equilibria have been demonstrated experimentally using TCP Reno and TCP Vegas/FAST in [25] for this example.

\section{B. Regular networks}

Examples 1 and 2 show that global uniqueness is generally not guaranteed in a multi-protocol network. We now show, however, that local uniqueness is basically a generic property of the equilibrium set. We present our main results on the structure of the equilibrium set here, and the proofs in the next subsection.

Consider an equilibrium price $p^{*} \in E$. Recall the active constraint set $\hat{L}$ defined by $p^{*}$. The equilibrium price $\hat{p}^{*}$ for the links in $\hat{L}$ is a solution of

$$
\hat{y}(\hat{p})=\hat{c}
$$

By the inverse function theorem, the solution of (20), and hence the equilibrium price $\hat{p}^{*}$, is locally unique if the Jacobian matrix $\hat{\mathbf{J}}\left(\hat{p}^{*}\right)=\partial \hat{y} / \partial \hat{p}$ is nonsingular at $\hat{p}^{*}$. We call a network $(c, m, R, U)$ regular if all its equilibrium prices are locally unique.

The next result shows that almost all networks are regular, and that regular networks have finitely many equilibrium prices. This justiffes restricting our attention to regular networks.

Theorem 4. Suppose assumptions A1 and A2 hold. Given any price mapping functions $m$, any routing matrix $R$ and utility futctions $U$,

1) the set of link capacities $c$ for which not all equilibrium prices are locally unique has Lebesgue measure zero in $\Re_{+}^{L}$.

2) the number of equilibria for a regular network $(c, m, R, U)$ is finite.

For the rest of this subsection, we narrow our attention to networks that satisfy an additional assumption:

A3: Every link $l$ has a single-link flow $(j, i)$ with $\left(U_{i}^{j}\right)^{\prime}\left(c_{l}\right)>0$.

Assumption A3 says that when the price of link $l$ is small enough, the aggregate rate through it will exceed its capacity. It implies that the active constraint set is unique and contains every link. Hence all results below that need A3 can be interpreted as with respect to a fixed active constraint set.

Since all the equilibria of a regular network have nonsingular Jacobian matrices, we can define the index $I(p)$ of $p \in E$ as

$$
I(p)= \begin{cases}1 & \text { if } \operatorname{det}(\mathbf{J}(p))>0 \\ -1 & \text { if } \operatorname{det}(\mathbf{J}(p))<0\end{cases}
$$

We have

Theorem 5. Suppose assumptions A1-A3 hold. Given any regular network, we have

$$
\sum_{p \in E} I(p)=(-1)^{L}
$$

where $L$ is the number of links.

Here, we give an important consequence of this theorem. Another implication to global uniqueness will be shown in Section V-A.

Corollary 6. Suppose assumptions Al-A3 hold. A regular network has odd number of equilibria.

Proof. Since both $I(p)$ and $(-1)^{L}$ are odd, the number of terms in the summation in Theorem 5 must be odd.

Notice that Corollary 6 implies the existence of equilibrium. Although we proved this in Section III in a more general setting, this simple corollary shows the power of Theorem 5.

Example 3: illustration of Theorem 5 and Corollary 6

We revisit Example 1 with modified utility functions. Recall that in Example 1, as $\epsilon$ varies from 0 to $1 / 24$, we trace out all 
equilibrium points. The components $x_{1}^{1}$ and $q_{1}^{1}=p_{1}^{1}$ of these equilibrium points are shown by the (red) solid line in Figure 3. Other sources $x_{i}^{j}$ and their effective end-to-end prices $q_{i}^{j}$ also lie on similar straight lines. Since the network has uncountably many equilibrium points, it is not regular. To make it regular, suppose we change the utility functions of sources $(j, i)$ to

$$
U_{i}^{j}\left(x_{i}^{j}, \alpha_{i}^{j}\right)= \begin{cases}\beta_{i}^{j}\left(x_{i}^{j}\right)^{1-\alpha_{i}^{j}} /\left(1-\alpha_{i}^{j}\right) & \text { if } \alpha_{i}^{j} \neq 1 \\ \beta_{i}^{j} \log x_{i}^{j} & \text { if } \alpha_{i}^{j}=1\end{cases}
$$

with appropriately chosen positive constants $\alpha_{i}^{j}$ and $\beta_{i}^{j}$. These utility functions can be viewed as a weighted version of the $\alpha$-fairness utility functions proposed in [20].

The basic idea of how to choose $\alpha_{i}^{j}$ and $\beta_{i}^{j}$ to generate only finitely many equilibrium points is as follows. First, we pick two points in the equilibrium set of Example 1, say, the points associated with $\epsilon=0.01$ and $\epsilon=0.04$. These choices of $\epsilon$ provide two distinct equilibrium points $(q, x)$ and $(\tilde{q}, \tilde{x})$. For instance, $\left(x_{1}^{1}, q_{1}^{1}\right)=(0.135,0.865)$ corresponds to $\epsilon=0.01$ and $(\tilde{q}, \tilde{x})=(0.165,0.835)$ corresponds to $\epsilon=0.04$, as illustrated in Figure 3 . Then, for each source $(j, i)$, find

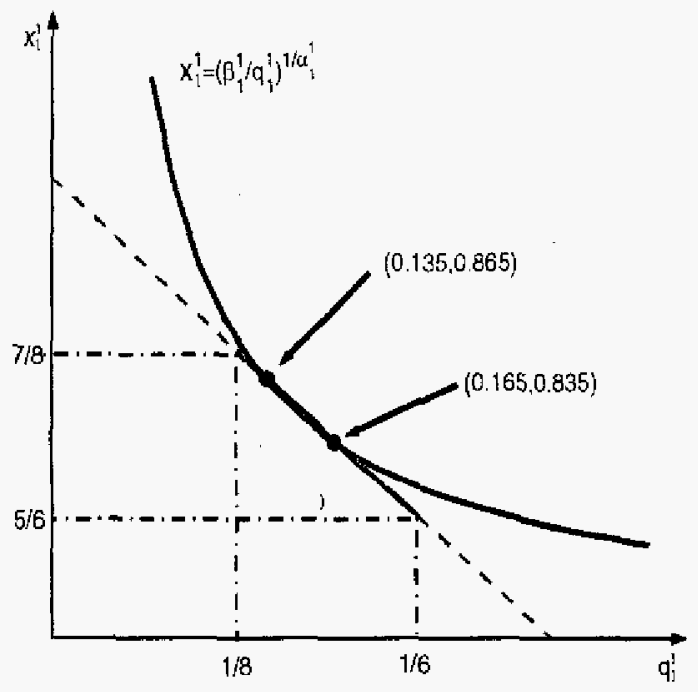

Fig. 3. Example 3: construction of multiple isolated equilibria.

$\alpha_{i}^{j}$ and $\beta_{i}^{j}$ such that (4) is satisfied by the two equilibrium points $\left(q_{i}^{j}, x_{i}^{j}\right)$ and $\left(\tilde{q}_{i}^{j}, \tilde{x}_{i}^{j}\right)$ with the new utility functions. This is illustrated in Figure 3 where relation (4) with the new utility function is represented by the (blue) curve, and $\alpha_{i}^{j}$, $\beta_{i}^{j}$ are chosen so that the curve passes through the original equilibrium points $\left(x_{1}^{1}, q_{1}^{1}\right)$ and $(\tilde{q}, \tilde{x})$. More specifically, given two equilibrium points $\left(q_{i}^{j}, x_{i}^{j}\right)$ and $\left(\tilde{q}_{i}^{j}, \tilde{x}_{i}^{j}\right)$, choose

$$
\begin{aligned}
\alpha_{i}^{j} & =\frac{\log \left(q_{i}^{j}\right)-\log \left(\tilde{q}_{i}^{j}\right)}{\log \left(\tilde{x}_{i}^{j}\right)-\log \left(x_{i}^{j}\right)} \\
\beta_{i}^{j} & =q_{i}^{j}\left(x_{i}^{j}\right)^{\alpha_{i}^{j}}
\end{aligned}
$$

The resulting $\alpha_{i}^{j}$ and $\beta_{i}^{j}$ for all flows $(j, i)$ are shown in Table I.

By construction, both $\left(p_{1}^{1}=0.135, p_{2}^{1}=0.230\right)$ and $\left(p_{1}^{1}=\right.$ $0.165, p_{2}^{1}=0.170$ ) are network equilibria. By Corollary 6 ,
TABLE 1

EXAMPLE 3: $\alpha_{i}^{j}$ AND $\beta_{i}^{j}$.

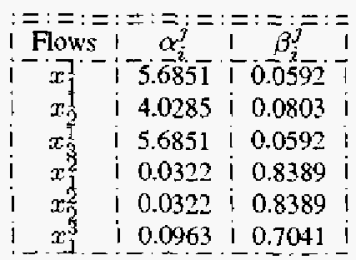

there is at least one additional equilibrium. Numerical search indeed located a third equilibrium with $p_{1}^{1}=0.142, p_{2}^{1}=$ 0.206 ).

We further check the local stability of these three equilibria under the gradient algorithm (23) to be introduced in Section IV-C. The eigenvalues and index for each equilibrium are shown in Table II. It turns out that the equilibrium $\left(p_{1}^{1}=\right.$ $0.142, p_{2}^{1}=0.206$ ) is not stable and has index 1 , while the other two are stable with index -1 . The dynamics of this

\begin{tabular}{|c|c|c|}
\hline $1 \overrightarrow{3} 5, \overline{0} . \overline{23}, 1 \overline{3} 5 \overline{-}$ & $\begin{array}{l}1 \\
-0.21-17.43-26.73\end{array}$ & \\
\hline$(0.142,0.206,0.142)$ & $0.21,-12.32,-22.40$ & 1 \\
\hline$(0.165,0.17,0.165)$ & $-12.41,-1.67,-0.67$ & \\
\hline
\end{tabular}

TABLE II

EXAMPLE 3: STABILITY AND INDICES OF EQUILIBRIA.

network under the gradient algorithm can be illustrated by a vectot filed. By symmetry, the equilibrium prices for the first and third link are always same. Therefore, we can draw the vector field restricted on the plane $p_{1}=p_{3}$ to illustrate the system dynamics. The phase portrait is shown in Figure 4 . The (red) dots represent the three equilibria. Note the equilibrium in the middle is a saddle point, and therefore unstable. The (red) arrows give the direction of this vector field. Individual trajectories are plotted with slim (blue) lines.

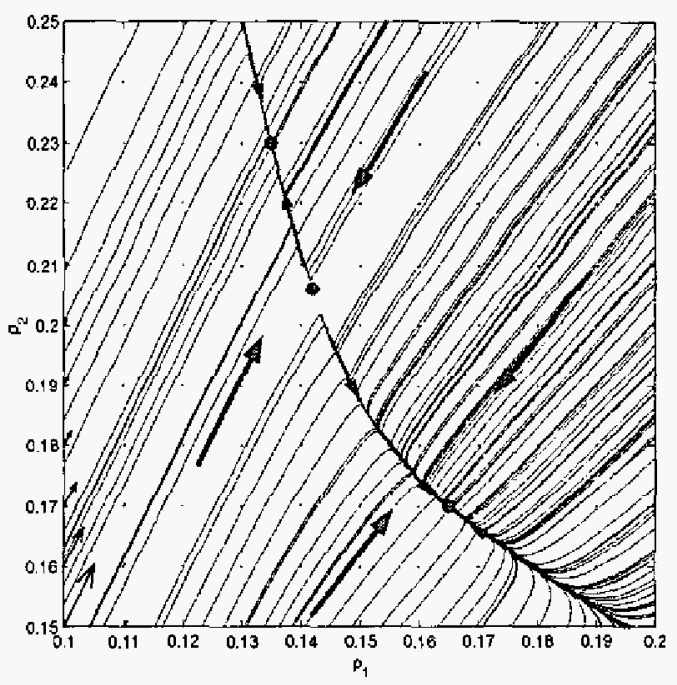

Fig. 4. Example 3: vector field of $\left(p_{1}, p_{2}\right)$. 


\section{Proofs and intuitions}

In this subsection we provide proofs for the results on local unliqueness.

Proof of Theorem 4. The main mathematical tool used in our proof is Sard's Theorem [4], [24], of which we quote a version here that is tailored to our problem. Let $G$ be an open subset of $s_{+}^{L}$ and let $F$ be a continuously differentiable function from $G$ to $\Re_{+}^{L}$. A point $y \in G$ is a critical point of $F$ if the Jacobian matrix $\partial F / \partial y$ of $F$ at $y$ is singular. A point $z \in \Re_{+}^{L}$ is a critical value of $F$ if there is a critical point $y \in G$ with $z=F(y)$. A point in $\Re_{+}^{L}$ is a regular value of $F$ if it is not a critical value.

Sard's theorem. If $F: G \rightarrow \Re_{+}^{L}$ is continuously differentiable on the open subset $G \subseteq \Re_{+}^{L}$, then the set of critical values of $F$ has Lebesgue measure zero in $\Re_{+}^{L}$.

Fix a routing matrix $R$ and utility functions $U$. There are at most $2^{L}-1$ different active constraint sets. Let $\hat{L} \subseteq L$ be such a combination with $\hat{L}$ links. Consider the set of all possible link capacities $c=\left(c_{l}, l \in L\right)$ under which the active constraint set is $\hat{L}$, i.e.. with such a capacity vector $c$, an equilibrium price $p$ has $p_{l}>0$ if $l \in \hat{L}$ and $p_{l}=0$ otherwise. Fix such an equilibrium point $p^{*}$. Again let $\hat{p}$ denote the price vector only for links in $\hat{L}$. Then $\hat{p}^{*}$ is not locally unique if the function $\hat{y}: \Re_{+}^{\hat{L}} \rightarrow \Re_{+}^{\hat{L}}$ defined by $\hat{y}(\hat{p})=\hat{R} x(\hat{p})$ has a singular Jacobian matrix $\partial \hat{y} / \partial \hat{p}$ at $\hat{p}^{*}$, i.e., if $\hat{p}^{*}$ is a critical point of $\hat{y}$. The set of such capacity vectors $\hat{c} \in \Re_{+}^{\hat{L}}$ under which all links in $\hat{L}$ have active constraints in equilibrium satisfy

$$
\hat{y}\left(\hat{p}^{*}\right)=\hat{c}
$$

and hence are critical values of $\hat{y}$. Since $\hat{y}$ is continuously differentiable by assumption A1, we can apply Sard's theorem and conclude that the set of such capacity vectors $\hat{c}$ has zero Lebesgue measure in $\Re_{+}^{\hat{L}}$. The extension to $\Re_{+}^{L}$ for all link capacities clearly also has zero Lebesgue measure in $\Re_{+}^{L}$.

Since we only have a finite number of different active constraint sets, the union of link capacity vectors that give rise to locally nonunique equilibria still has zero Lebesgue measure. This proves the first part of the theorem.

The equilibrium set $E$ defined in (7) is closed because $y(p)$ is continuous, and is bounded by Lemma 1 . Hence $E$ is compact. Since $(c, m, R, U)$ is a regular network, every $p \in E$ is locally unique, i.e., for each $p \in E$ we can find an open neighborhood such that it is the only equilibrium in that open set. The union of these open sets forms a cover for set $E$. Since $E$ is compact, it admits a finite subcover [15], i.e., $E$ can be covered by a finite number of open sets each containing a single equilibrium. Hence, the number of equilibria is finite.

Proof of Theorem 5. By assumption A3, we can always find $p_{\min }>0$ such that for any price $p$ and link $l$ with $p_{l}<p_{\min }$, we have

$$
\sum_{j} \sum_{i} R_{l i}^{j} x_{i}^{j}(p)>c_{l}
$$

Let $G:=\left[p_{\min }, p_{\max }\right]^{L}$ where $p_{\max }$ is defined in Lemma 1 . Clearly, all equilibria are in the set $G$. To prove our result, we will invoke a version of the Poincare-Hopf Index Theorem tailored to our problem [27], [18].

Poincare-Hopf Index Theorem. Let $D$ be an open subset of $\Re$ and $v: D^{L} \rightarrow \Re^{L}$ be a smooth vector field, with nonsingular Jacobian matrix $\partial v / \partial p$ at every equilibrium. If there is a $G \subseteq$ $D^{L}$ such that every trajectory moves inward of region $G$, then the sum of the indices of the equilibria in $G$ is $(-1)^{L}$.

Gradient project algorithm. To construct the vector field $v$ required by the index theorem, let $D^{L}=G$ and consider the following gradient algorithm from $G$ to $G$ proposed in [13]. The prices are updated at time $t$ according to

$$
\dot{p}(t)=\Lambda(R x(t)-c)
$$

where $\Lambda>0$ is an $L \times L$ diagonal matrix whose elements represent stepsizes. A source updates its rate based on the end-to-end price

$$
x(t)=x(p(t))
$$

A consequence of assumption $A 3$ is that $p(t) \geq p_{\min }>0$ for all $t$ under the gradient algorithm (21)-(22). This guarantees a unique active constraint set that is $L$. Hence the equilibrium set $E$ defined in (7) is equivalent to $E=\left\{p \in \Re_{+}^{L_{f}} \mid y(p)-c=0\right\}$.

Combining (21)-(22) with $y(p(t))=R x(t)$ yields the required vector field $v$ :

$$
\dot{p}(t)=\Lambda(y(p(t))-c)=: v(p(t))
$$

whose Jacobian matrix is:

$$
\frac{\partial v(p)}{\partial p}=\Lambda \mathbf{J}(p)=\Lambda \frac{\partial y(p)}{\partial p}
$$

where $\mathbf{J}(p)$ is given by ( 8 ). Clearly, $p^{*}$ is an equilibrium point of $v$, i.e., $v\left(p^{*}\right)=0$, if and only if $p^{*}$ is a network equilibrium, i.e., $p^{*} \in E$. Since the retwork $(c, m, R, U)$ is regular, $\mathbf{J}(p)$ is nonsingular at every network equilibrium $p^{*} \in E \subset G$. Since $\Lambda$ is a positive diagonal matrix, $\partial v(p) / \partial p$ is also nonsingular by (24) at all its equilibrium points $p$ in $G$, as the index theorem requires.

Consider any point $p$ on the boundary of $G$. For any $l$, we have one of two cases:

1) If $p_{l}(t)=p_{\max }$, link $l$ will be underutilized, $y_{l}(p(t))<$ $c$, and $\dot{p}_{l}<0$ according to (23).

2) If $p_{l}(t)=p_{\min }$, the aggregate rate at link $l$ will exceed $c_{l}, y_{l}(p(t))>c_{l}$, and $\dot{p}_{l}>0$ according to (23).

Therefore, every point $p$ on the boundary of $G$ will move inward. Hence our result directly follows from the PoincareHopf index theorem.

\section{SUFFCIENT CONDITIONS FOR GLOBAL UNIQUENESS}

The exact condition under which network equilibrium is globally unique is generally hard to prove. This section provides four sufficient conditions for global uniqueness. The first condition relates local stability of the equilibria to their uniqueness. The second condition generalizes the full rank condition of $R$ from single-protocol network to multi-protocol 
network. The third condition guarantees uniqueness when the price mapping functions are linear and link-independent. The final condition implies global uniqueness of linear networks.

\section{A. First condition: local stability and global uniqueness}

Recall that under assumption A3, we can assume without loss of generality that $L$ is the unique active constraint set that contains all links. We say an equilibrium $p^{*} \in E$ locally stable if the corresponding Jacobian matrix $\mathbf{J}\left(p^{*}\right)$ defined in (8) is stable, that is, every eigenvalue of $\mathbf{J}\left(p^{*}\right)=\partial y\left(p^{*}\right) / \partial p$ has negative real part. Hence a locally stable $p^{*}$ is also locally unique, but the converse may not hold. To justify this definition, take the matrix $\Lambda$ in the gradient algorithm (23) to be $\lambda I$. Then the linearized system is ( $\delta p$ denotes the perturbation around $p^{*}$ ):

$$
\delta \dot{p}=\lambda \mathbf{J}\left(p^{*}\right) \delta p
$$

Since $\lambda>0$ is a scalar, the real part of the eigenvalues of $\lambda \mathbf{J}\left(p^{*}\right)$ has the same sign as that of the eigenvalues of $\mathbf{J}\left(p^{*}\right)$. Hence the local asymptotic stability of $p^{*}$ implies that the gradient algorithm (23) converges locally to $p^{*}$.

Theorem 7. Suppose assumptions $A 1-A 3$ hold. If all equilibria have index $(-1)^{L}$, then $E$ contains exactly one point. In particular, if all equilibria are locally stable, then $E$ contains exactly one point.

Proof: The first claim of the theorem directly follows from Theorem 5. We now claim that an equilibrium $p^{*} \in E$ which is locally stable has an index $I\left(p^{*}\right)$ of $(-1)^{L}$. To prove the claim, consider a locally stable equilibrium price $p^{*}$. All the eigenvalues of $\mathbf{J}\left(p^{*}\right)$ have negative real parts. Moreover, since $\mathbf{J}\left(p^{*}\right)$ has real entries, complex eigenvalues come in conjugate pairs. The determinant of $\mathbf{J}\left(p^{*}\right)$ is the product of all its eigenvalues. If there are $k$ conjugate pairs of complex eigenvalues and $L-2 k$ pairs of real eigenvalues, the product of all eigenvalues has the same sign as $(-1)^{L-2 k}$ which has the same sign as $(-1)^{L}$. Hence the index of a locally stable equilibrium is $(-1)^{L}$.

This result may seem surprising on the first sight as it relates the local stability of an algorithm to the uniqueness property of a network. This is because both equilibrium and local stability are defined in terms of the function $y(p)$ : an equilibrium $p^{*}$ satisfies $y\left(p^{*}\right)=c$ and the local asymptotic stability of $p^{*}$ is determined by $\partial y\left(p^{*}\right) / \partial p$. The connection between these two properties is made exact by the index theorem. An implication of this result is that if there are multiple equilibria, then no algorithm $\dot{p}=f(p(t))$, whose linearization around each equilibrium $p^{*} \in E$ satisfies $\partial f\left(p^{*}\right) / \partial p=\partial y\left(p^{*}\right) / \partial p$, can ever be found to locally stabilize all of the equilibria. Conversely, if we can find a region that contains $E$ and such an algorithm such that i) all boundary points of that region move inwards, and ii) the algorithm locally stabilizes any equilibrium, then there is exactly one network equilibrium.

Local stability can be checked in several ways. For example, if the Jacobian matrix is diagonal dominant at an equilibrium, then the equilibrium is locally stable. This test of local stability will be used in Theorem 11 below.

\section{B. Second condition: negative definiteness of $J(p)$}

In a single-protocol network, for the equilibrium price to be unique, it is sufficient that the routing matrix $R$ has full row rank. Otherwise, only the source rates are unique, not necessarily the link prices. In a multi-protocol network, this is no longer sufficient. We now provide another sufficient condition that plays the same role in a multi-protocol network as the rank condition on $R$ does in a single-protocol network (see also the remark after the proof of Theorem 9).

Let $f=\left(f_{1}, \ldots, f_{n}\right)$ be a vector of real-valued functions defined on $\Re^{n}$. Let $G:=\left\{z \in \Re \Re^{n} \mid f(z)=0\right\}$ and $\operatorname{co} G$ be its convex hull. Define a set $V(G)$ of vectors as

$$
V(G):=\{v \mid v=\phi-\psi \text { for } \psi, \phi \in \operatorname{co} G\}
$$

as a function of the set $G$.

Lemma 8. If for every $z \in$ coG, the Jacobian matrix $\boldsymbol{J}(z)=$ $\partial f(z) / \partial z$ exists and $v^{T} \boldsymbol{J}(z) v<0$ for all $v \in V(G)$, then $G$ contains at most one point.

Proof. For the sake of contradiction, assume there are two distinct points $\phi$ and $\psi$ in $G$ such that $f(\phi)=f(\psi)=0$. Let

$$
g(\theta):=\phi+\theta(\psi-\phi) \text { where } \theta \in[0,1]
$$

Then

$$
\frac{d f(g(\theta))}{d \theta}=\mathbf{J}(g(\theta)) \frac{d g(\theta)}{d \theta}=\mathbf{J}(g(\theta))(\psi-\phi)
$$

Hence,

$$
f(\psi)-f(\phi)=\int_{0}^{1} \mathbf{J}(g(\theta))(\psi-\phi) d \theta
$$

Multiplying both sides by $(\psi-\phi)^{T}$ yields

$$
\begin{array}{r}
(\psi-\phi)^{T}(f(\psi)-f(\phi))= \\
\int_{0}^{1}(\psi-\phi)^{T} \mathbf{J}(g(\theta))(\psi-\phi) d \theta
\end{array}
$$

The left hand-side of the above equation is 0 , and the righthand side is negative under the assumption of the theorem. This contradiction proves the theorem.

Let $f=y$, and let $G=E$ be the set of network equilibria. Then Lemma 8, together with Theorem 2, provides a sufficient condition for global uniqueness of network equilibrium.

Theorem 9. Suppose assumptions $A 1-A 3$ hold. If for every price vector $p \in c o E$, the Jacobian matrix $J(p)$ defined in (8) exists and $v^{T} J(p) v<0$ for all $v \in V(E)$, then there exists a globally unique network equilibrium.

In the single-protocol case, a similar result has been obtained in [20]. However, for that case, the Jacobian matrix is negative definite when $R$ has full row rank. Then the condition in Theorem 9 always holds and the equilibrium is unique. In the multi-protocol case, the Jacobian matrix is in general not symmetric and hence not negative definite. Therefore $R$ having full row rank is no longer sufficient for the condition in the theorem to hold.

Since we do not know the equilibrium set $E$, the condition in the theorem cannot be directly applied to prove global 
uniqueness. To use the theorem, however, it is sufficient to find a convex superset $\tilde{E}$ of $E$ and a superset $\tilde{V}$ of $V(E)$ such that $v^{T} \mathbf{J}(p) v<0$ for all $p \in \tilde{E}$ and $v \in \tilde{V}$. This implies the condition in Theorem 9 and hence global uniqueness. We illustrate this procedure in the next example.

\section{Example 4: application of Theorem 9 to verify global uniqueness}

We visit Example I for the third time but using log utility functions for all sources, i.e.,

$$
U_{i}^{j}\left(x_{i}^{j}\right)=\log \left(x_{i}^{j}\right) \text { for all }(j, i)
$$

Let the Jacobian matrix be

$$
\mathbf{J}(p)=\left[\begin{array}{lll}
J_{11} & J_{12} & J_{13} \\
J_{21} & J_{22} & J_{23} \\
J_{31} & J_{32} & J_{33}
\end{array}\right]
$$

where $J_{k l}=J_{k l}(p)$ are functions of prices $p$ given by (8). For example

$$
J_{\Sigma 1}=-\frac{1}{p_{1}^{2}}-\frac{5}{\left(5 p_{1}+p_{2}\right)^{2}}-\frac{1}{\left(p_{1}+p_{3}+3 p_{2}\right)^{2}}
$$

It can be seen that $\mathbf{J}(p)$ is not negative definite for general $p$ unlike in the single-protocol case. Even though $E$ can be hard to find, we demonstrate how to find a simple convex superset $\tilde{E}$ of $E$ and a simple superset $\tilde{V}$ of $V(E)$.

Consider the convex set

$$
\tilde{E}:=\left\{p \in \Re_{+}^{3} \mid 1 \leq p_{1}=p_{3} \leq 2,1 \leq p_{2} \leq 2\right\}
$$

We claim that $E \subseteq \tilde{E}$. To see this, let $p$ be an equilibrium price. If $p_{1}<1$, then $x_{1}^{1}=1 / p_{1}$ will exceed the link capacity 1 , and hence $p_{1} \geq 1$. A similar argument gives $p_{2} \geq 1$. To see $p_{1} \leq 2$, assume it is not true. Then

$$
\begin{aligned}
& x_{1}^{1}=1 / p_{1}<1 / 2 \\
& x_{1}^{2}=1 /\left(5 p_{1}+p_{2}\right)<1 / 11 \\
& x_{1}^{3}=1 /\left(2 p_{1}+3 p_{2}\right)<1 / 7
\end{aligned}
$$

Summing them yields $x_{1}^{1}+x_{1}^{2}+x_{1}^{3}<1$. Hence the network is not in equilibrium, contradicting that $p$ is an equilibrium price. Hence $p_{1} \leq 2$. The argument for $p_{2} \leq 2$ is similar.

Using the definition of $\tilde{E}$, we can bound all $J_{k l}(p)$ for $p \in$ $\tilde{E}$. The results are collected in Table III.

TABLE III

EXAMPLE 4: BOUNDS ON ELEMENTS OF J $(p)$

\begin{tabular}{|c|c|c|}
\hline Elements & Upperbound & Lowerbound \\
\hline$J_{11}$ & -0.2947 & -1.1789 \\
$J_{22}$ & -0.2939 & -1.1756 \\
$J_{33}$ & -0.2947 & -1.1789 \\
$J_{23}$ & -0.0447 & -0.1789 \\
$J_{32}$ & -0.0369 & -0.1478 \\
$J_{12}$ & -0.0369 & -0.1478 \\
$J_{21}$ & -0.0447 & -0.1789 \\
$J_{13}$ & -0.0100 & -0.0400 \\
$J_{31}$ & -0.0100 & -0.0400 \\
\hline
\end{tabular}

Let

$$
\tilde{V}:=\left\{v \in \Re_{+}^{3} \mid v_{1}=v_{3}\right\}
$$

We claim that $V(E) \subseteq \tilde{V}$. To show this, note that $\operatorname{co} E \subseteq \tilde{E}$ since $c o E$ is the smallest convex set that contains $E$. Hence $V(E) \subseteq V(\tilde{E})$. Since $p_{1}=p_{3}$ at equilibrium, $v_{1}=v_{3}$ holds for any $v \in V(\tilde{E})$ from the definition of $\tilde{E}$. Hence, $V(\tilde{E}) \subseteq \tilde{V}$ and therefore $V(E) \subseteq \tilde{V}$.

We now check that $v^{T} \mathbf{J}(p) v<0$ for all $p \in \tilde{E}$ and $v \in \tilde{V}$. For any $v \in \tilde{V}, v^{T} \mathbf{J}(p) v$ is the following quadratic form in $v_{1}$ and $v_{2}$ :

$$
\begin{aligned}
v^{T} \mathbf{J}(p) v= & v_{1}^{2}\left(J_{11}+J_{33}+J_{13}+J_{31}\right)+ \\
& v_{1} v_{2}\left(J_{12}+J_{21}+J_{23}+J_{32}\right)+v_{2}^{2} J_{22}
\end{aligned}
$$

If $v_{1}$ and $v_{2}$ have the same signs, then since $J_{k l}$ are all negative from Table III, $v^{T} \mathbf{J}(p) v<0$. If $v_{1}$ and $v_{2}$ have opposite sign, then a sufficient condition for $v^{T} \mathrm{~J}(p) v<0$ is

$$
\left(J_{12}+J_{21}+J_{23}+J_{32}\right)^{2}<4 J_{22}\left(J_{11}+J_{33}+J_{13}+J_{31}\right)
$$

Using Table III, it is easy to check that the maximum value of $\left(J_{12}+J_{21}+J_{23}+J_{32}\right)^{2}-4 J_{22}\left(J_{11}+J_{33}+J_{13}+J_{31}\right)$ is -0.2895 . Therefore we have found a superset $\tilde{E}$ of $\operatorname{co} E$ and a superset $\tilde{V}$ of $V(E)$ such that $v^{T} \mathbf{J}(p) v<0$ for all $p \in \tilde{E}$ and all $v \in \dot{V}$. This implies the condition of Theorem 9 and hence the global uniqueness of network equilibrium.

\section{Third condition: linear link-independent $\mathrm{m}^{j}$}

When the price mapping functions are linear and linkindependent, i.e., $m_{l}^{j}\left(p_{l}\right)=k^{j} p_{i}$ for some scalar $k^{j}>0$, it is easy to show that we have an unusual situation in the theory of heterogeneous protocols where the equilibrium rate vector $x$ solves the following concave maximization problem

$$
\max _{x} \sum_{i, j} k^{j} U_{i}^{j}\left(x_{i}^{j}\right) \quad \text { s. t. } R x \leq c
$$

Therefore, such a network always has a globally unique equilibrium when $U_{i}^{j}$ are strictly concave.

Here we provide another proof using Theorem 9.

Theorem 10. Suppose assumptions $A 1-A 3$ hold and $R$ has full row rank. If for all $j$ and $l, m_{l}^{j}\left(p_{l}\right)=k^{j} p_{l}$ for some scalar $k^{j}>0$, then there is a unique network equilibrium.

Proof. We prove this by showing that the Jacobian matrix $J(p)$ defined in $(8)$ is negative definite over all $p \geq 0$. Then the result follows from Theorem 9 .

Under the assumptions of the theorem, $\mathbf{J}(p)$ can be simplified into (from (8)-(10))

$$
\begin{aligned}
\mathbf{J}(p) & =\sum_{j} R^{j} D^{j}(p)\left(R^{j}\right)^{T} \frac{\partial m^{j}(p)}{\partial p} \\
& =\sum_{j} k^{j} R^{j} D^{j}(p)\left(R^{j}\right)^{T}
\end{aligned}
$$

where $D^{j}(p)=\partial x^{j}(p) / \partial q^{j}$. Since $U_{i}^{j}$ are strictly concave, $D^{j}(p)$ is a strictly negative diagonal matrix for all $p \geq 0$, Now, $J(p)$ is symmetric. Moreover, since $R$ has full row rank, $R R^{T}$ is positive definite, i.e., for any nonzero vector $v \in \Re^{L}$,

$$
\sum_{j} v^{T} R^{j}\left(R^{j}\right)^{T} v=\sum_{j}\left(\left(R^{j}\right)^{T} v\right)^{T}\left(R^{j}\right)^{T} v>0
$$


Then there exists at least one $j$ such that $\eta^{j}:=\left(R^{j}\right)^{T} \hat{v}$ is nonzero. Without lose of generality, assume it is $j=1$. Then

$$
\begin{aligned}
v^{T} \mathbf{J}(p) v & =v^{T} \sum_{j} k^{j} R^{j} D^{j}(p)\left(R^{j}\right)^{T} v \\
& =\sum_{j} k^{j}\left(\eta^{j}\right)^{T} D^{j}(p) \eta^{j} \\
& \leq k^{1}\left(\eta^{1}\right)^{T} D^{1}(p) \eta^{1}<0
\end{aligned}
$$

where the first inequality follows from the fact that $D^{j}(p)$ is negative definite. Hence $\mathbf{J}(p)$ is negative definite.

\section{Fourth condition: linear networks}

We now apply Theorem 7 to prove global uniqueness of linear networks. Consider the classic line network shown in Figure 5. There are $L$ links and $L+1$ flows. Suppose without

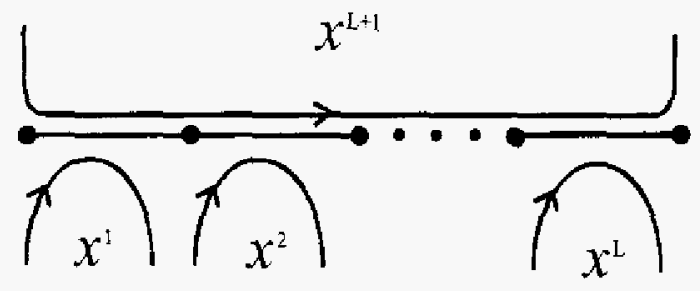

Fig. 5. Corollary 11: line network.

loss of generality that every flow uses a different protocol. This implies that $D^{j}(p)=\partial x_{j}(p) / \partial q^{j}$ is a negative scalar under assumption A1. Denote by $e^{j}$ a $L \times 1$ vector with 1 in the $j$ th entry and 0 elsewhere, and 1 a $L \times 1$ vector with 1 in every entry. Then $R^{j}=e^{j}$ for $j=1 \ldots L$, and $R^{L+1}=\mathbf{1}$.

Theorem 11. Suppose assumptions A1-A2 hold. The linear network in Figure 5 has a unique equilibrium.

Proof. Take $\Lambda=I$ in the gradient algorithm (23). We will prove that all the eigerivalues of the Jacobian matrix

$$
\mathbf{J}(p)=\sum_{j} R^{j} D^{j}(p)\left(R^{j}\right)^{T} \frac{\partial m^{j}(p)}{\partial p}
$$

have negative real part for all $p \geq 0$. This implies that all equilibria are locally stable. By Theorem 7 there must be a unique equilibrium.

In the network shown in Figure 5, for $j=1 \ldots L$,

$$
\left(R^{j}\right)^{T} \frac{\partial m^{j}(p)}{\partial p}=\frac{\partial m_{j}^{j}(p)}{\partial p_{j}}\left(e^{j}\right)^{T}
$$

Since $D^{j}(p)$ is a negative scalar, we can define a positive number $\beta_{j}$ such that:

$$
R^{j} D^{j}(p)\left(R^{j}\right)^{T} \frac{\partial m^{j}(p)}{\partial p}=-\beta_{j} e^{j} \cdot\left(e^{j}\right)^{T}
$$

For $j=L+1, \partial m^{j}(p) / \partial p$ is a positive definite diagonal matrix. Recall that $D^{j}(p)$ is a scalar. Assume that the $i$ th diagonal entry of matrix $D^{j}(p) \partial m^{j}(p) / \partial p$ is $-\gamma_{i}$. Denote by $\gamma$ the $L \times 1$ veciors formed from $\gamma_{i}$. Then for $j=L+1$ :

$$
R^{j} D^{j}(p)\left(R^{j}\right)^{T} \frac{\partial m^{j}(p)}{\partial p}=-\mathbf{1} \cdot \mathbf{1}^{T} \operatorname{diag}\left(\gamma_{i}\right)=-\mathbf{1} \gamma^{T}
$$

By combining the results above, we obtain

$$
\begin{aligned}
\mathbf{J}(p) & =\sum_{j=1}^{L+1} R^{j} D^{j}\left(R^{j}\right)^{T} \frac{\partial m^{j}}{\partial p} \\
& =-\sum_{1}^{L} \beta_{j} e^{j} \cdot\left(e^{j}\right)^{T}-\mathbf{1} \gamma^{T} \\
& =-\operatorname{diag}\left(\beta_{i}\right)-\mathbf{1} \gamma^{T}
\end{aligned}
$$

By the following Lemma, all the eigenvalues of above matrix have negative real parts. Therefore, there must be a unique equilibrium by Theorem 7 .

Lemma 12. Suppose that $B$ is a positive definite diagonal matrix, and $\gamma$ is a positive vector, then the eigenvalues of $B+\boldsymbol{I} \gamma^{T}$ have positive real parts.

Proof: See Appendix VII-B.

\section{Remarks:}

1) The above result can be generalized to include more than one multi-hop flows, provided they all belong to the same type $L+1$ and the sets of links they traverse form an inclusion. i.e., $L\left(x_{1}^{L+1}\right) \supseteq L\left(x_{2}^{L+1}\right) \supseteq \cdots \supseteq$ $L\left(x_{n}^{L+1}\right)$ for $n$ multi-hop flows.

2) Theorem 7 also implies the global uniqueness of equilibrium for any network in which no flow passes through more than 2 links in the active constraint set, when A1A3 hold. In this case, the Jacobian matrix $\mathbf{J}(p)$ is strictly diagonally dominant with negative diagonal entries, and hence its determinant is $(-1)^{L}$. As a consequence, we need at least a three-link network to have multiple equilibrium if $\mathrm{Al}-\mathrm{A} 3$ are satisfied.

3) If $\mathrm{A} 3$ is violated, then there are two-link networks that have multiple equilibria; see Example 5 with two links and two protocols in Appendix VII-A. Since uniprotocol network always has a unique equilibrium, Example 5 is a "smallest" network that has non-unique equilibria.

\section{CONCLUSION AND Future work}

When sources sharing the same network react to different pricing signals, the current duality model no longer explains the equilibrium of bandwidth allocation. We have introduced a mathematical formulation of network equilibrium for multiprotocol networks and studied several fundamental properties, such as existence, local uniqueness, number of equilibria, and globai uniqueness. We prove that equilibria exist, and are almost always locally unique. The number of equilibria is almost always finite, must be odd when they are associated with the same active constraint set. We provide four sufficient conditions for global uniqueness.

Acknowledgments: We thank Kim Border and John Ledyard of Caltech, and Ramesh Johari of Stanford for useful discussions, and R. Srikant of UIUC for some references. This work is performed as part of the FAST Project supported by NSF, ARO, AFOSR and Cisco. We also thank the support from 
NSF Grants CCF-0440443, CNS-0417607, CNS-0427677, and SISL Fellowship.

\section{REPERENCES}

[1] K. Arrow and F. Hahn. General Competitive Analysis. Holden-Day, 1971.

[2] T. Basar and G. Olsder. Dynomic Noncooperative Game Theory, 2nd Edtion. SIAM. 1999.

[3] G. Debreu. Theory of Value. New York:Wiley. 1959.

[4] G. Debreu. Economies with a finite set of equilibria. Econometrica, 38(3): 387-392. May, 1970.

[5] G. Debreu. Economic Theory in the Mathematical Mode. Noble Memorial lecture, 8 December, 1983.

[6] E. Dierker. Two Remarks on the Number of Equilibria of an Econony. Econometrica, $40(5)$ : 751-753, Sep, 1972.

[7] E. Dierker and H. Dierker. The Local Uniqueness of Equilibria. Econontetrica, 40(5): 867-881. Sep, 1972.

[8] C. Jin, D. Wei, and S. Low. TCP FAST: motivation, architecture, alaorithms, performance. Proceedings of IEEE Infocom 2004.

[9] F. Kelly, A. Maoulloo, and D. Tan. Rate control for conmunication networks: shadow prices, proportional faimess and stability. Journal of she Operational Research Sociefy. 49:237-252.1998.

[10] S. Kunniyur and R. Srikant. End-to-end congestion conirol: utility functions, tandom losses and ECN marks. IEEE/ACM Transactions on Networking. 11(5):689-702. Oct 2003.

[11] K. Kurata. G. Hasegawa, and M. Murata. Fairness Comparisons between TCP Reno and TCP Vegas for Future Deployment of TCP Vegas. Proceedings of INET 2000.

[12] S. Low. A Duality Model of TCP and Quewe Management A]gorithms. IEEE/ACM Trans. on Networking, 1 1(4):525-536. August 2003.

[13] S. Low and D. Lapsley. Optimization flow control. I: basic algotithm and convergence. IEEEACM Transactions on Networking, 7(6):861874. December 1999.

[14] S. Low, F. Paganini, and J. Doyle. Internet Congestion Control. IEEE Control Systems Magzine. February 2002.

[15] J. Marsden and M. Hoffman. Elementary Classical Analysis. Second edition. W.H.Freeman and Company. New York 1993.

[16] A. Mas-Colell. The Theory of General Economic Equilibrium. A Differentiable Approach. Cambridge University Press, 1985.

[17] A. Mas-Colell, M. Whinston and J. Green. Microeconomic Theory, Oxford University Press, 1995.

[18] J. Milnor. Topology from the Differentiable Vewpoint. The Uimiversity Press of Virginia, Charlottesville, 1972

[19] J. Mo, R. La. V. Anantharam, and J. Walrand. Analysis and Comparison of TCP Reno and Vegas. Proceedings of IEEE lifocom, March 1999.

[20] J. Mo and J. Waltand. Fair End-to-End Window-Based Congestion Control. IEEE/ACM Transactions on Networking, 8(5):556-567, Od 2000 .

[21] M. Osborne and A. Rubinstein. A Course in Game Theory. The MIT Press, 1994.

[22] I. Pearce and J. Wise. On the Uniqueness of Competitive Equilibrium: Part 1, Unbounded Demand. Econometrica,41(5): 817-828, Sep, 1973.

[23] I. Pearce and J. Wise. On the Uniqueness of Competitive Equilibrium: Part II, Bounded Demand. Econometrica.42(5): 921-932. Sep, 1974.

[24] S. Sternberg. Lectures on Differential Geometry. Prentice-Hall, 1964.

[25] A. Tang, J. Wang, S. Hedge and S. Low. Equilibrium and Fairness of Networks Shared by TCP Reno and FAST. submitted, 2005.

[26] A. Tang, J. Wang and S. Low. Is fair allocation always inefficient. Proceedings of IEEE Infocom 2004.

[27] H. Varian. A Third Remark on the Number of Equilibria of an Economy Econometrica, 43(5/6):985-986, Sep-Nov, 1975 .

[28] H. Yaiche, R. R. Mazumdar, and C. Rosenherg. A game theoretic framework for bandwidth allocation and pricing in broadband networks. IEEE/ACM Transactions on Networking, 8(5). October 2000.

\section{APPENDIX}

\section{A. Smallest network with multiple equilibria}

Example 5: a two-link network with non-unique equilibria

In this example, we again assume that all sources use the same utility function defined as

$$
U_{i}^{j}\left(x_{i}^{j}\right)=-\frac{1}{2}\left(1-x_{i}^{j}\right)^{2}
$$

The network topology is shown in Figure 6 with link capacities $c=[1,1]$. The corresponding routing matrices for these two

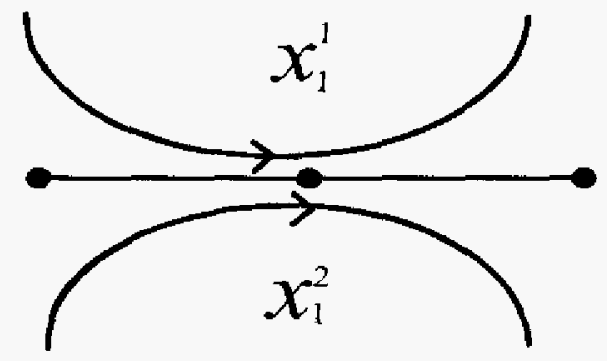

Fig. 6. Example 5: a netwotk with 2 links and 2 protocols.

protocols are

$$
R^{1}=R^{2}=\left[\begin{array}{l}
1 \\
1
\end{array}\right]
$$

We use linear price mapping functions $m^{j}(p)=K^{j} p, j=$ 1,2 , where $K^{j}$ are $2 \times 2$ matrices given by

$$
K^{1}=I, \quad K^{2}=\operatorname{diag}(1,3)
$$

As for Example 1, we check the matrix

$$
\sum_{i=1}^{2} R^{i}\left(R^{i}\right)^{T} K^{i}=\left[\begin{array}{ll}
1 & 4 \\
1 & 4
\end{array}\right]
$$

which has determinant 0 , implying multiple equilibria. It is easy to verify that the following points are all equilibria:

$$
p_{1}=\epsilon, \quad p_{2}=1 / 4-\epsilon / 2, \quad \text { where } \in \in[0,1 / 2]
$$

The corresponding rates are:

$$
x_{1}^{1}=3 / 4-\epsilon / 2, \quad x_{1}^{2}=1 / 4+\epsilon / 2
$$

The capacity constraints are all tight.

Remarks: Note that even with a single protocol, the example above has non-unique equilibrium price vectors since the routing matrix is not full rank. However, in that case, the equilibrium rates are unique, unlike the case of multiple protocols.

\section{B. Proof for Lemma 12}

Proof. Suppose that $\lambda$ is an eigenvalue of $B+1 \gamma^{T}$, then $\operatorname{diag}\left(\beta_{i}-\lambda\right)+1 \gamma^{T}$ is singular. If $\lambda=\beta_{i}$ for certain $i$, then, since $\beta_{i}>0, \lambda$ is positive. Otherwise the following matrix is also singular

$$
I+\operatorname{diag}\left(\frac{1}{\beta_{i}-\lambda}\right) 1 \gamma^{T}
$$

The rank of matrix $\operatorname{diag}\left(1 /\left(\beta_{i}-\lambda\right)\right) \mathbf{1} \gamma^{T}$ is 1 . Moreover it has only one nonzero eigenvalue equal to $\sum_{i} \gamma_{i} /\left(\beta_{i}-\lambda\right)$. For the matrix in (28) to be singular, it must have a zero eigenvalue, and this is possible if and only if

$$
\sum_{i} \frac{\gamma_{i}}{\beta_{i}-\lambda_{i}}=-1
$$

The real part of $\gamma_{i} /\left(\beta_{i}-\lambda_{i}\right)$ is $\gamma_{i}\left(\beta_{i}-\operatorname{Re} \lambda\right) /\left|\beta_{i}-\lambda\right|^{2}$. If Re $\lambda \leq 0$, the sum of the real part of $\gamma_{i} /\left(\beta_{i}-\lambda_{i}\right)$ cannot be -1 . So we must have $\operatorname{Re} \lambda>0$. 